www.jmscr.igmpublication.org

Index Copernicus Value: 79.54

ISSN (e)-2347-176x ISSN (p) 2455-0450

crossrefDOI: https://dx.doi.org/10.18535/jmscr/v7i2.120

\title{
Scimitar Syndrome: A Rare Cause for Dyspnea
}

\author{
Authors \\ Dr Rohit Thakare ${ }^{1}$, Dr Sarvesh Prajapati ${ }^{2}$, Dr B C Kalmath ${ }^{3}$ \\ Department of Cardiology, Bombay Hospital Institute of Medical Sciences, Mumbai \\ ${ }^{1}$ Email: rohit.thakare111@gmail.com
}

\begin{abstract}
Scimitar syndrome or pulmonary veno lobular syndrome is a rare complex cardio-pulmonary anamoly characterised by partial anamolous pulmonary venous connection. It can present as infant or adult form. We present a case of adult female evaluated for breathlessness had mild right heart dilatation and turned out to be adult form of scimitar syndrome, along with brief review of literature.

Keywords-Scimitar syndrome, Anamolous pulmonary venous connection, rare cuase of dyspnea.
\end{abstract}

\section{Introduction}

Scimitar syndrome (SS) is a rare complex variable congenital anamoly with an estimated incidence of approximately 2 in 100000 births. It is characterised by anomalous pulmonary venous drainage from the right lung to the inferior vena cava. ${ }^{1}$ The associated findings include right lung hypoplasia, abnormal arterial supply with or without sequestration, cardiac defects like ASD. Infantile and adult form of the disease has been noted, prognosis being good for adult form. Adult patient may develop heart failure, repeated respiratory infections A typical chest radiograph shows Turkish sword sign, however further imaging is required to confirm diagnosis. Surgical correction is recommended for patients with a pulmonary-tosystemic blood flow ratio exceeding 2:1 because of the risk of development of pulmonary hypertension and right ventricular failure ${ }^{2}$

\section{Case Report}

A 38 year old female with no comorbidities presented with complaints of easy fatiguability, breathlessness on exertion NYHA grade 2 since two months associated with intermittent palpitations. She denied history of recurrent respiratory tract infections. She was hemodynamically stable and clinical examination was normal. Her routine blood investigations were within normal limits. Ecg showed incomplete RBBB with notching of qrs in inferior leads. CXR was almost normal. 2D Echo revealed mild dilatation of right atrium and right ventricle, normal LV function however no evidence of atrial septal defect and no PH. Subsequently CT pulmonary angiography done which showed following findings-

Right upper and lower lobar pulmonary vein were seen to form a common vertical vein draining into infradiaphragmatic portion of IVC, measuring $1.6 \mathrm{~cm}$ in diameter extending for approx. length 3 $\mathrm{cm}$, left pulmonary veins drained normally into left atrium, dilatation of IVC \& Hepatic veins were 
noted. Hence diagnosis of partial anamolous pulmonary venous drainage, Scimitars syndrome adult variant was made. Patient also underwent cardiac catheterisation to quantify shunt $\mathrm{Qp} / \mathrm{Qs}$ ratio. Oxymetry samples showed significant step up in saturation at low RA level and Qp/Qs was 1.7 suggestive of moderate left to right shunt. She responded to diuretics and was kept on medical line of management and advised regular follow up.

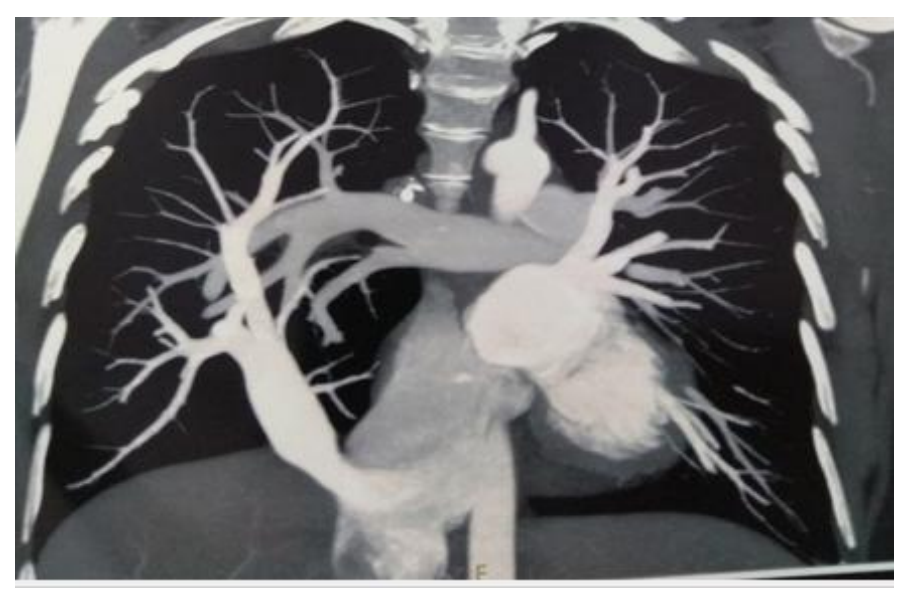

Fig 1: CT pulmonary angiography in levo phase showing anamolous drainage of right lung pulmonary veins into IVC

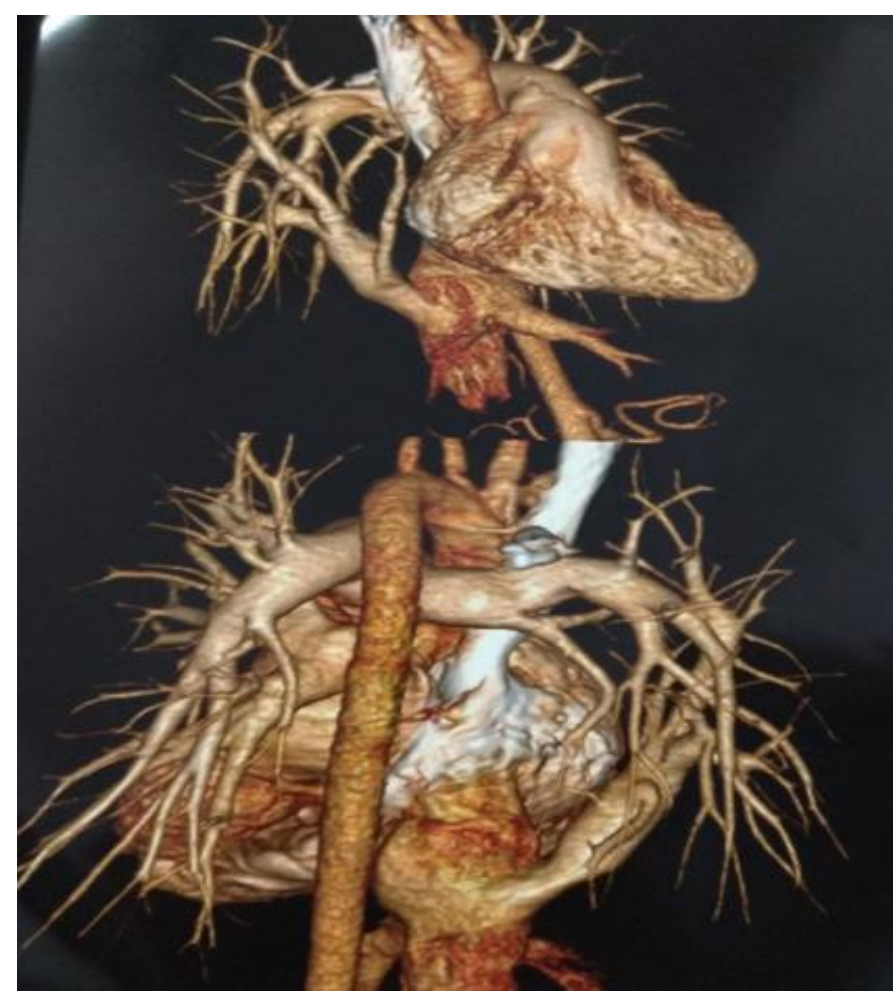

Fig 2: $3 \mathrm{D}$ reconstruction $\mathrm{CT}$ images showing anamolous drainage of right pulmonary veins into IVC.

\section{Discussion}

Scimitar syndrome is rare congenital anamoly of lung with partial anamolous pulmonary venous drainageto right atrium, coronary sinus, Inferior Vena Cava (IVC), or hepatic circulation with reported incidence of 1-3 cases per 100000 live births. It shows female preponderance and behaves functionally like ASD. Associated anamolies include right lung hypoplasia, dextraposition of heart, and anomalous systemic arterial supply from aorta or one of its branches to the right lung. Rarely, a scimitar syndrome of the left lung has been reported. There are three variants of scimitar syndrome: an infantile form which is diagnosed before one year and is associated with heart failure and pulmonary hypertension, an adult form with milder symptoms, and a form associated with other congenital heart malformations. This syndrome has varied presentations, from an asymptomatic state ${ }^{3}$ to severe pulmonary hypertension and/or heart failure. ${ }^{4}$ The presentation varies as per age group. Sometimes it is associated with cardiac anomalies like Atrial Septal Defect (ASD), Ventricular Septal Defect (VSD), Patent Ductus Arteriosus (PDA), tetralogy of Fallot, coarctation, hypoplastic left heart or endocardial cushion defects, diaphragmatic hernia, and anomalies of the bony thorax.Partial Anomalous Pulmonary Venous Return (PAPVR) has a prevalence of $0.4-0.7 \%$ in the general population, with prevalence abruptly rising in patients with atrial septal defects $(10-15 \%$ in ostiumsecundum type and nearly $100 \%$ in sinus venosus type).

This anomaly results in left-to-right shunt, with partial admixture of deoxygenated and oxygenated blood. However this shunt is usually hemodynamically insignificant, with most patients being asymptomatic or mildly symptomatic. As such, it is commonly detected incidentally on imaging studies. Clinical symptomatology of the scimitar syndrome depends on the age at which the patient presents. Infantile variant has got worse outcome and infant having scimitar syndrome present with cyanosis, poor growth, $\mathrm{PAH}$, and often complex cardiac defects, many of them need 
surgical intervention having a high mortality. ${ }^{5}$ Conversely, the 'adult' form remains asymptomatic for many years and has a much more favourable outcome. $^{6}$

The severity of symptoms depends on the degree of the shunt present and may be manifested as dyspnea on exertion, palpitations, syncope, or congestive heart failure. Our case was adult variety of Scimitar syndrome and had exertionaldyspnea and palpitations. Though rare, supraventricular arrhythmias are associated with this entity.

Radiologically the shadow extends from the lateral superior position of right lung to cardiophrenic angle. The appearance closely resembles that of Turkish sword or Scimitar. ${ }^{7}$ Two forms of "scimitar veins" have been described in literature. 1. Partial or complete right pulmonary vein draining into IVC 2 . Both right upper and lower pulmonary vein drainig to IVC as in our case. Even Cine MRI and 3D contrast enhanced MR angiography provides detail analysis of anamolous pulmonary venous return.

There is no consenses for indication of surgery in adult variant especially when patient is asymptomatic or mildly symptomatic. Some authors suggest surgical intervention in those cases who land up in congestive failure or reistant to medical therapy with large left to right shunt i.e. shunt usually more than $2: 1$ and those with lung sequestration causing repeated lung infections. ${ }^{8}$ Surgical correction aims to direct blood from schimitar vein to left atrium, ligation /embolization of vascular supply to the sequestered lung lobe.

\section{Conclusion}

We present case of adult variant of schimitar syndrome who was breathless on exertion with atypical presentation, had no recurrent respiratory tract infections. A high index of suspicion is reuired to diagnose shunts when right heart is mildly dilated in a symptomatic patient. The left to right shunt was moderate on cardiac catheterization .Hence she was treated with medical line of management. She responded well to diuretics and has been advised regular follow up.

\section{References}

1. Cooper G. Case of malformation of the thoracic viscera consisting of imperfect development of the right lung and transposition of the heart. London Med Gaz 1836;18:600-1

2. Najm HK, Williams WG, Coles JG et al. Scimitar syndrome: twenty years' experience and results of repair. $\mathbf{J}$ Thorac Cardiovasc Surg 1996;112:1161-9. 10.1016/S0022-5223(96)70129-0

3. Khan A, Ring NJ, Hughes PD. Scimitar syndrome (congenital pulmonary venolobar syndrome) Postgrad Med J. 2005;8:216

4. Mordue BC. A case series of five infants with scimitar syndrome. Adv Neonatal Care.

5. Huddleston CB, Exil V, Canter CE, Mendeloff EN. Scimitar syndrome presentingin infancy. Ann Thorac Surg 1999;67:154-9

6. Gudjonsson U, Brown JW. Scimitar syndrome. Semin Thorac Cardiovasc Surg Pediatr Card Surg Annu2006;9:5662.10.1053/j.pcsu.2006.02.011

7. Neill CA, Ferenca C, Sabiston DC. The familial occurrence of hypoplastic right lung with systemic arterial supply and venous return-Scimitar syndrome. Bull Johns Hopkins Hosp. 1960;107:1 